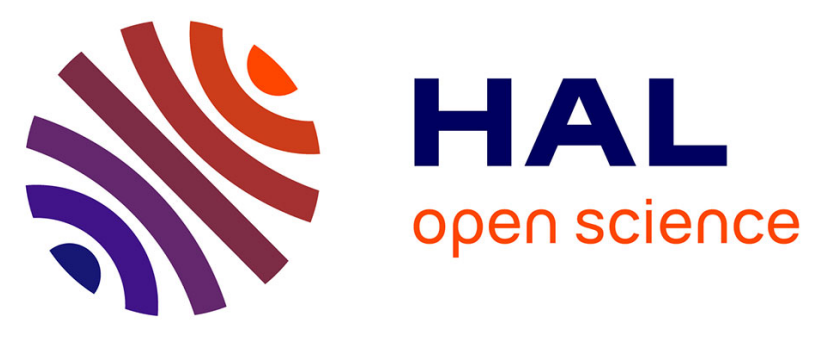

\title{
Influence of screw configuration, residence time, and specific mechanical energy in twin-screw extrusion of polycaprolactone/multi-walled carbon nanotube composites
}

\author{
Tobias Villmow, Bernd Kretzschmar, Petra Pötschke
}

\section{To cite this version:}

Tobias Villmow, Bernd Kretzschmar, Petra Pötschke. Influence of screw configuration, residence time, and specific mechanical energy in twin-screw extrusion of polycaprolactone/multiwalled carbon nanotube composites. Composites Science and Technology, 2010, 70 (14), pp.2045.

10.1016/j.compscitech.2010.07.021 . hal-00682264

\section{HAL Id: hal-00682264 \\ https://hal.science/hal-00682264}

Submitted on 24 Mar 2012

HAL is a multi-disciplinary open access archive for the deposit and dissemination of scientific research documents, whether they are published or not. The documents may come from teaching and research institutions in France or abroad, or from public or private research centers.
L'archive ouverte pluridisciplinaire HAL, est destinée au dépôt et à la diffusion de documents scientifiques de niveau recherche, publiés ou non, émanant des établissements d'enseignement et de recherche français ou étrangers, des laboratoires publics ou privés. 


\section{Accepted Manuscript}

Influence of screw configuration, residence time, and specific mechanical energy in twin-screw extrusion of polycaprolactone/multi-walled carbon nanotube composites

Tobias Villmow, Bernd Kretzschmar, Petra Pötschke

PII:

S0266-3538(10)00290-3

DOI:

10.1016/j.compscitech.2010.07.021

Reference:

CSTE 4775

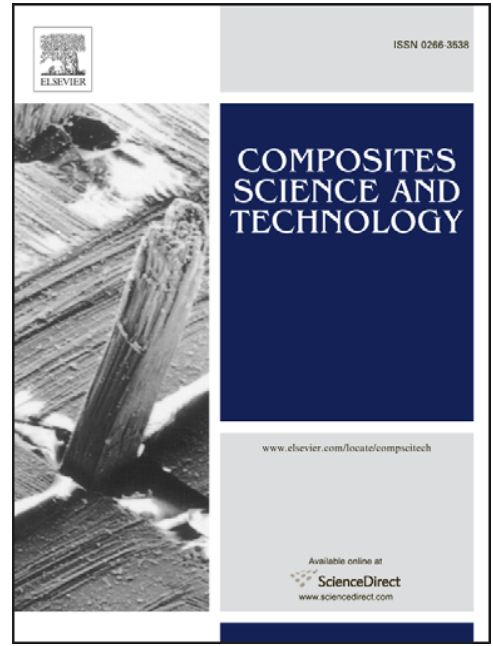

To appear in:

Composites Science and Technology

Received Date:

1 April 2010

Revised Date:

20 July 2010

Accepted Date:

22 July 2010

Please cite this article as: Villmow, T., Kretzschmar, B., Pötschke, P., Influence of screw configuration, residence time, and specific mechanical energy in twin-screw extrusion of polycaprolactone/multi-walled carbon nanotube composites, Composites Science and Technology (2010), doi: 10.1016/j.compscitech.2010.07.021

This is a PDF file of an unedited manuscript that has been accepted for publication. As a service to our customers we are providing this early version of the manuscript. The manuscript will undergo copyediting, typesetting, and review of the resulting proof before it is published in its final form. Please note that during the production process errors may be discovered which could affect the content, and all legal disclaimers that apply to the journal pertain. 
Influence of screw configuration, residence time, and specific mechanical energy in twinscrew extrusion of polycaprolactone/multi-walled carbon nanotube composites

Tobias Villmow, Bernd Kretzschmar, Petra Pötschke*

Leibniz Institute of Polymer Research Dresden, Hohe Strasse 6, 01069 Dresden, Germany

\section{Abstract}

Melt processing of thermoplastic-based nanocomposites is the favoured route to produce electrically conductive or electrostatic dissipative polymer composites containing carbon nanotubes (CNT). As these properties are desired at low filler fractions, a high degree of dispersion is required in order to benefit from the intrinsic CNT properties. This study discusses the influence of screw configuration, rotation speed, and throughput on the residence time and specific mechanical energy (SME) and the resulting macroscopic CNT dispersion in polycaprolactone (PCL) based masterbatches containing $7.5 \mathrm{wt}$ \% multi-walled carbon nanotubes (MWNT) using an intermeshing co-rotating twin-screw extruder Berstorff ZE25.

The processing conditions were found to have a strong influence on the residence time $\left(t_{R}\right)$ of the extrudates and on the CNT dispersion within the masterbatches as assessed using light microscopy. Both, an increase of rotation speed and throughput resulted in a decrease of $t_{R}$ whereas the use of back-conveying elements and the extension of the processing length showed the opposite effect. As the increase of rotation speed results in higher SME inputs a significant increase of CNT dispersion was found, whereas an increase of throughput resulted in worse dispersion. Beside these machine parameters, the design of the screw can further promote the CNT dispersion especially when using distributive screw configurations containing mixing elements. The lowest area fraction of undispersed primary MWNT agglomerates within PCL masterbatches was found when using an extended distributive screw having a length to diameter ratio L/D of 48 instead of 36 . The few remaining macroscopic agglomerates could be entirely dispersed in a subsequent masterbatch dilution process resulting in a very low electrical percolation threshold of 0.24 vol.\% MWNT.

Keywords: $\quad$ A. Carbon nanotubes, Nano composites, Polymer-matrix composites (PMCs); D. Optical microscopy; E. Extrusion

* Corresponding author. Tel.: +49/3514658395; fax: +49/3514658565.

E-mail address: poe@ipfdd.de (P. Pötschke) 


\section{Introduction}

A favoured strategy for the development of new functional polymer materials is the mixing of a conventional matrix polymer with further polymer components (polymer blends) and/or solid fillers (composites). The big advantage of this route and such composite materials is based on the unlimited number of possible property combinations, much better conditions for the market launch, and higher costumer acceptance in comparison to new homopolymers. Carbon nanotubes (CNT) are one of these functional fillers. After their first detailed description in scientific literature in 1991 [1] they got into the focus of research due to their outstanding electrical [2-4], thermal [5-7], and mechanical properties [8-10]. These excellent characteristics predestine them as filler for polymer-based composites, although a transfer of properties to the polymer matrix is challenging, as CNT tend to form strong agglomerates resulting from physical interactions like van-der-Waals forces. Possible physical entanglements of high-aspect ratio CNT yielding from the synthesis process often further enhance the strength of primary agglomerates. These primary agglomerates have to be dispersed and distributed within the polymer in order to benefit from the properties of individual CNT. Recently, a correlation between dispersability of different CNT materials in aqueous solutions and resulting composite properties after incorporation of these CNT into a polymer matrix via melt mixing was published by Krause et al. [11]. A correlation between CNT dispersability in an aqueous surfactant solution, quantified by means of a LUMiFuge stability analyser, dispersion in the melt and the resulting percolation thresholds of these CNT in polyamide 6.6 was found. The higher the CNT dispersability, the lower percolation thresholds were observed.

A high degree of CNT dispersion within composite materials is desired with regard to their applications, as undispersed primary CNT agglomerates cause problems during processing when blocking melt filters during extrusion and spinning. Further, mechanical properties will be affected negatively, as macroscopic CNT agglomerates remaining in the final composite part act as imperfection under mechanical load. A third aspect requiring high quality of CNT dispersion are the electrical properties, especially the percolation threshold, which is connected to the available amount of individual CNT contributing to the percolation network. Thus, in order to get to electrically conductive composites the amount of expensive CNT can be reduced significantly when achieving high degrees of CNT dispersion. In this context, also nanoarrangement of nanotubes plays an important role, but first requisition for achieving electrically conductive networks is the dissolution of primary nanotube agglomerates. 
Depending on the polymers nature, different processing techniques like high-shear mixing, solution casting, in-situ polymerisation, the latex approach, and melt processing are discussed in literature. Melt processing is the preferential method for the processing of thermoplasticbased composites and has the highest relevance for industrial applications, as it is based on conventional industrial processing technologies like twin-screw extrusion or injection moulding. Even if some knowledge was gained about the influence of processing conditions on composite properties in small-scale processing [12-14] so far only a limited number of publications deal with industrial relevant melt processing techniques. For the influence of twin-screw extrusion conditions on nanotube dispersion and electrical resistivity first results based on polylactic acid (PLA) are presented in [15] indicating that high rotation speed still ensuring a certain residence time of the melt combined with a screw profile containing mainly mixing elements are favourable to disperse and distribute the CNT in the PLA matrix. From investigations regarding the processing conditions during the shaping processes injection moulding $[16,17]$ and compression moulding $[12,14]$ it was concluded that secondary agglomeration of individualized CNT is important in order to create electrically percolated networks within composites. The process of secondary agglomeration, however, requires well-dispersed nanotubes, which have to be generated during the composite production step. In order to produce suitable amounts of composite materials for injection moulding [16-19] dilution of commercially available [16] or self-made masterbatches [18, 19] as well as directly mixed composites [17] were used.

In comparison to small-scale mixing, the melt mixing using continuous processes reveals new challenges. The residence time of the polymer melt during twin-screw extrusion is a complex function of rotation speed, throughput, and screw configuration and cannot be adjusted independently like in case of discontinuous small-scale mixing equipments, e.g. microcompounders. The interaction between different processing parameters leads to complex functions of shear conditions and residence time, both affecting CNT dispersion. The modular assembly of most twin-screw extruders enables the use of different processing unit length (L/D) and especially the extrusion screw can be varied in nearly endless configurations. The basis for every extrusion screw is the feeding zone followed by the melting zone, which consists of kneading elements ensuring the melting of the polymer pellets. The following metering zone enables the mass transport by conveying elements. Within that zone the dispersion and distribution of the filler and the homogenisation of the melt are realised by kneading and mixing elements. The former promote the break-up of 
cohesive agglomerates, while the latter increase the homogeneity of the mixture by generating new interfacial area.

Measurable extrusion characteristics are needed in order to find relationships between the extrusion process and the composite properties. For this reason, the residence time and specific mechanical energy (SME) were determined in order to interpret the observed phenomena regarding the processing dependent macroscopic CNT dispersion. Since residence time distribution (RTD) curves could not be recorded, as the extruded unfilled polycaprolactone (PCL) could not be collected as a continuous strand, the minimum residence time (or dead time) was determined for all experiments. It was assumed that the RTD curves have similar shape and thus the minimum residence time can be used as characteristic for the residence time $t_{R}$. As second parameter for the characterisation of the extrusion process, the SME was chosen that is the amount of mechanical energy, which is dissipated as heat inside the material during the extrusion process. Usually SME increases with increasing viscosity, increasing screw speed, and decreasing throughput. Furthermore, the processing temperature affects the SME as it influences the polymer viscosity.

As method to determine the macroscopic CNT dispersion in PCL based masterbatches transmission light microscopy (LM) was chosen ensuring a relatively large investigated sample volume. The use of LM enables the detection of the undispersed fraction of primary CNT agglomerates remaining within the polymer after melt processing. In comparison, transmission electron microscopy (TEM) analyses the dispersed CNT fraction in just very small sample volumes, is not able to illustrate big remaining primary agglomerates, and as it is illustrated in [15] it is difficult to draw conclusions on dispersion differences in highly loaded masterbatches. Scanning electron microscopy represents the CNT dispersion in the near of the surface of fractured or cut samples and no global information about the nanotube dispersion within the volume can be extracted. Therefore, light microscopy was the preferred method for the presented study.

A few publications report about the small-scale processing and properties of nanocomposites based on CNT and PCL. It was reported that this filler increases the thermomechanical properties like storage and loss modulus enabling a broader range of application for this thermoplastic [20]. Two different approaches of nanocomposite processing like melt compounding [21] and in-situ polymerisation [22] are described to result in percolated network structures within the PCL matrix at MWNT contents of around $2.0 \mathrm{wt} . \%$. A significantly lower percolation threshold of $0.08 \mathrm{wt}$.\% CNT was discussed for PCL/SWNT 
composites, which were prepared by solution mixing with the aid of a zwitterionic surfactant [23].

The basis for this study are recently published results on the influence of twin-screw extrusion conditions on the dispersion of CNT in a polylactic acid matrix, where a set of two screw configurations and a variation of rotation speed was used to produce masterbatches containing 7.5 and 15.0 wt.\% CNT [15] by means of a Berstorff ZE 25 co-rotating twin-screw extruder. The results indicated that the quality of nanotube dispersion within the masterbatch is deciding for the dispersion in the therefrom diluted composites. Therefore, this study focuses on the achievement of a low amount of remaining primary agglomerates in masterbatches. It was also shown, that a nanotube content of $7.5 \mathrm{wt} \%$ is very suitable in order to investigate the influence of extrusion conditions, as the use of higher amounts restricts the range of possible variations especially concerning the rotation speed. Thus, this article discusses the influence of the extrusion conditions screw configuration (5 different screws), throughput $(5,10$, and 15 $\mathrm{kg} / \mathrm{h}$ ), and rotation speed (100 and $500 \mathrm{rpm}$ ) on the macroscopic CNT dispersion within PCL masterbatches. The residence time $t_{R}$ and specific mechanical energy SME are used as process characteristics and are correlated with the macroscopic CNT dispersion analysed by means of light microscopy.

\section{Materials and methods}

\subsection{Materials}

Polycaprolactone (PCL) CAPA 6800 (Perstorp, U.K.) is a biodegradable polyester with a low melting point of around $60^{\circ} \mathrm{C}$ and a glass transition temperature of about $-60^{\circ} \mathrm{C}$. The grade CAPA 6800 is a homopolymer with a molecular weight of $80.000 \mathrm{~g} / \mathrm{mol}$ and a MFI of $3 \mathrm{~g} / 10 \mathrm{~min}\left(2.16 \mathrm{~kg}\right.$ at $\left.160^{\circ} \mathrm{C}\right)$. The frequency dependent viscosity at $210^{\circ} \mathrm{C}$, presented as a master curve based on measurements at 150,180 , and $210^{\circ} \mathrm{C}$, and the zero shear viscosity are shown in Figure 1. The PCL having a density of $1.14 \mathrm{~g} / \mathrm{cm}^{3}$ was melt mixed with Nanocyl ${ }^{\circledR}$ N7000 (Nanocyl S.A., Belgium), which are thin multi-walled carbon nanotubes (MWNT) with a purity of $90 \%$ produced via the catalytic carbon vapour deposition process. The nanotubes have a typical diameter of $9.5 \mathrm{~nm}$ and an average length of $1.5 \mu \mathrm{m}$. The density of MWNT after incorporation into a polymer is approximately $1.75 \mathrm{~g} / \mathrm{cm}^{3}$ [24]. A small amount of as-received CNT was placed on an adhesive carbon tape mounted on a SEM sample holder in order to perform a SEM study. An Ultra plus microscope (Carl Zeiss, Germany) was used and the samples were not sputter coated. 


\subsection{Methods}

\subsubsection{Composite preparation via twin-screw extrusion}

\section{Processing of PCL/CNT masterbatches}

In order to investigate the influence of twin-screw extrusion conditions, several PCL/CNT masterbatches containing $7.5 \mathrm{wt}$ \% CNT (N7000) were produced under variation of screw configuration, rotation speed, and throughput. The temperature profile was kept constant on a relatively high level (Table 1) to ensure the processability of PCL for all parameter combinations. The compounding was performed using an intermeshing co-rotating twinscrew extruder ZE25 (Berstorff, Germany) having a screw diameter of $25 \mathrm{~mm}$ and barrel lengths of $900 \mathrm{~mm}(\mathrm{~L} / \mathrm{D}=36)$ or $1200 \mathrm{~mm}(\mathrm{~L} / \mathrm{D}=48)$. PCL pellets and the powdery MWNT N7000 material were fed simultaneously into the hopper by gravimetric dosing.

Berstorff provides extrusion screws with a modular assembly build of individual screw elements, like conveying (1,2), back-conveying (3), kneading (4-6), and mixing elements $(7,8)$ which are shown in Figure 2. The screw element characteristics are presented in Table 2. The conveying elements are characterised by their length, pitch, and number of flights. Kneading and mixing elements need further specification for their number of discs, staggering angle (kneading), and number of cogs (mixing). Berstorff designates conveying elements with "L" and back-conveying elements with " $R$ ". The design of the screws is based on two screw configurations recently published by our group [15]. This set of screws was extended to five different screws (SC1-SC5, see Figure 3) to get more information about the influence of screw configuration on the residence time and CNT dispersion within the PCL matrix. The two dispersive screw configurations SC1 and SC2 contain kneading and conveying elements in the metering zone. The number and position of kneading elements was kept constant, whereas the number of back-conveying elements was increased for SC2 to enhance the residence time of the extrudates. The kneading blocks consisted of five kneading discs with a positive $\left(45^{\circ}\right)$ staggering angle. Both screws were designed to have a length to diameter ratio of 36 .

Three distributive screw configurations containing mixing and conveying elements were designed according to the dispersive ones with respect to the position of mixing elements. The conveying elements of SC1 were maintained and the kneading elements within the mixing zone were substituted by mixing elements to create SC3. Similarly to the idea behind SC2, the screw configuration of SC4 was again designed to have additional back-conveying elements. The fifth screw configuration was designed based on SC4 as a distributive mixing screw, but having an extended L/D of 48 instead of 36. Furthermore, this screw contains additional 
mixing elements. The mixing elements had two or three discs, whereas each disc was equipped with ten cogs.

\section{Processing of PCL/CNT composites by masterbatch dilution and electrical testing}

The masterbatch exhibiting the highest degree of CNT dispersion was diluted with neat PCL under optimised conditions (SC5, $500 \mathrm{~min}^{-1}$, and $5 \mathrm{~kg} / \mathrm{h}$ ) to produce composites with 0.125 , $0.25,0.375,0.5,0.75,1.0,1.5,2.0,3.0$, and $4.0 \mathrm{wt} . \%$ MWNT in order to determine the percolation behaviour of MWNT within the PCL matrix. For extrusion, masterbatch pellets and pure PCL were premixed prior feeding into the hopper.

The volume resistivity of the composites was determined according to the standards ASTM D 4496 and ASTM D 257 on compression-moulded plates. Plates having a diameter of $65 \mathrm{~mm}$ and a thickness of $0.5 \mathrm{~mm}$ were prepared from the pelletised materials using a PW $40 \mathrm{EH}$ hot press (Paul-Otto Weber GmbH, Germany). The pellets were first molten within the pressing frames using PTFE foils as release material at $220^{\circ} \mathrm{C}$ for $2 \mathrm{~min}$. The following compression step was performed with a pressing speed of $6 \mathrm{~mm} / \mathrm{min}$ at a pressing force of $20 \mathrm{kN}$ for $3 \mathrm{~min}$. The pressed plates were cooled down at room temperature. The resistivity measurements were performed at room temperature. A Keithley 8009 Resistivity Test Fixture combined with a Keithley 6517A Electrometer was used to determine the electrical resistance of highly resistive samples on the circular plates. In case of low resistance samples, small strips $\left(30 \times 3 \mathrm{~mm}^{2}\right)$ were cut from these plates and electrical resistance was measured using a self-made four-point test fixture in combination with a Keithley multimeter Model 2000. Volume resistance $R_{v}$ was converted to volume resistivity $\rho_{v}$ using Equation 1, where A is the effective area of the sample involved in the measurement and $t$ is the specimen thickness.

$\rho_{\mathrm{v}}=\mathrm{R}_{\mathrm{v}} \cdot \frac{\mathrm{A}}{\mathrm{t}}[\Omega \mathrm{cm}] \quad$ Equation 1

\subsubsection{Determination of residence time $t_{R}$}

Residence time distribution (RTD) curves where a tracer concentration is plotted versus time could not be recorded, as the extruded unfilled PCL could not be collected as a continuous strand, although the CNT filled masterbatches could be collected properly. Classical RTD curves show a sharp increase of tracer concentration after passing the dead time $t_{D}$ followed by a maximum and tail. For this study, five granules of PCL based masterbatches $(7.5 \mathrm{wt} . \%$ $\mathrm{CNT}$ ) were used as tracer (colourant) enabling an optical detection of $t_{\mathrm{D}}$. This time is characterized by a fast increase of tracer concentration leading to a high colour contrast from opaque to black. After reaching steady state extrusion conditions of the neat PCL, the 
masterbatch granules were fed to the hopper in a pulsed manner and the determined dead time as one characteristic value of the RTD curve was used in the following as residence time $t_{R}$.

\subsubsection{Specific mechanical energy}

The specific mechanical energy (SME) is the amount of mechanical energy can be calculated using Equation 2, where the torque $\tau[\mathrm{kJ}]$, rotation speed $\mathrm{N}\left[\mathrm{s}^{-1}\right]$, and throughput $\dot{\mathrm{m}}[\mathrm{kg} / \mathrm{s}]$ are involved.

$$
\mathrm{SME}=\frac{\tau \cdot \mathrm{N}}{\dot{\mathrm{m}}}\left[\frac{\mathrm{kJ}}{\mathrm{kg}}\right]
$$

\section{Equation 2}

Specifically, it is the work input from the drive motor into the material being extruded and thus provides a good characterisation of the extrusion process. Usually SME increases with increasing viscosity, increasing screw speed, and decreasing throughput. Furthermore, the processing temperature affects the SME as it influences the polymer viscosity. Both the extrusion operation and the screw performance can be well characterised by the SME value. It is also an important parameter in extrusion processes, as it provides a measurable parameter which can be correlated with the quality characteristics of the final product. In case of the presented study, SME values were correlated with the macroscopic CNT dispersion.

\subsubsection{Determination of macroscopic CNT dispersion}

\section{Macroscopic CNT dispersion of extruded materials}

The state of macroscopic CNT dispersion by considering remaining primary CNT agglomerates within the PCL masterbatches was investigated using transmission light microscopy on thin sections of extruded pellets, which were prepared following the standard ISO 18553. Thin sections were microtomed (JUNG RM 2055 microtome, Leica, Germany) from at least six masterbatch pellets with a histo-diamond knife (Diatome, Switzerland) at cryo conditions $\left(-40^{\circ} \mathrm{C}\right)$ and a thickness of $2.5 \mu \mathrm{m}$. The thin sections of the composite pellets with low CNT amounts (diluted samples) were cut with thicknesses between 15 and $20 \mu \mathrm{m}$. The micrographs were imaged with a BH2 microscope (Olympus, Germany) using an objective with a 10x magnification. A particle analysis using the software ANALYSIS (Olympus, Japan) was performed in order to quantify the area fraction $\mathrm{A}_{\mathrm{A}}$ of undispersed primary $\mathrm{CNT}$ agglomerates according to Equation 3, where $\mathrm{A}_{\mathrm{CNT}}$ is the area occupied by remaining primary $\mathrm{CNT}$ agglomerates and $\mathrm{A}_{0}$ the overall section area. According to the ISO 18553 standard, agglomerates with diameters smaller than $5 \mu \mathrm{m}$ were neglected. Furthermore, from the area of primary CNT agglomerates the circle-equivalent diameter was 
calculated. The resulting CNT agglomerate distribution curves are discussed in context with processing conditions.
$\mathrm{A}_{\mathrm{A}}=\frac{\mathrm{A}_{\mathrm{CNT}}}{\mathrm{A}_{0}} \cdot 100 \%[\%]$

\section{Equation 3}

\section{Development of macroscopic CNT dispersion along extrusion length}

As the ZE25 extruder enables the removal of the extrusion screw, in addition investigations of the development of CNT dispersion in PCL along the length of the screw during extrusion were performed. Thus, further information concerning the impact of different mixing and kneading elements on the CNT dispersion could be obtained. During mixing PCL with 7.5 wt.\% CNT, the extruder was stopped after reaching steady state extrusion conditions and the screws were pulled out from the processing unit within a few seconds. Several samples of approximately one gram were taken from the screws (using screw configurations SC2 and SC5, rotation speed $500 \mathrm{rpm}$ and throughput $5 \mathrm{~kg} / \mathrm{h}$ ) behind every mixing or kneading element and used for light microscopic investigations.

\section{Results and discussion}

\subsection{Morphology of the MWNT material}

A SEM study of the MWNT N7000 raw material was carried out to investigate the initial primary agglomerate size and structure. Both parameters are important to judge the state of dispersion after incorporation into the PCL matrix and to estimate the inherent dispersability of the nanotubes. Although nanotubes are nanofillers, the as delivered N7000 material appears in the shape of macroscopic primary agglomerates with diameters up to several millimetres. A typical SEM micrograph illustrating these agglomerated structures with a relatively broad particle size distribution is shown in Figure 4-A. Besides the strongly pronounced agglomeration, the SEM study revealed that $\mathrm{N} 7000$ form rope-like structures, whereas these ropes are built by a large number of individual CNT (Figure 4-B and C), which are packed in a relatively loose way (Figure 4-D). As the interspace between individual CNT is quite large, the dispersability of this CNT material should be high, as it was confirmed in aqueous surfactant dispersions and PA6.6 melts by Krause et al. [11].

\subsection{Influence of processing conditions on residence time}

When dispersing any kind of filler in a polymeric matrix, mixing time is a crucial factor to control the filler dispersion. Especially the "erosion mechanism" described for carbon black in a Newtonian fluid is time depending and follows a first order rate [25], as erosion takes place from the agglomerate surface and is relatively slow compared to the "rupture 
mechanism", where big agglomerates are fragmented into a small number of relatively large agglomerates. Based on this model, Kasaliwal et al. developed a new model for CNT based composites, which enables the calculation of the share of erosion or rupture contributing to the dispersion process of CNT [26]. An important input parameter for these considerations is the mixing time.

Many publications dealing with melt mixing of polymer/CNT composites discuss the influence of mixing time on the CNT dispersion when using microcompounders as mixing device [e.g. 13, 27]. These microcompounders are discontinuous mixers, where rotation speed and mixing time can be set up independently, whereas the screw configuration is generally fixed. In case of twin-screw extrusion the residence time is a complex factor, which is a function of rotation speed, throughput, and screw configuration. In order to find relationships between the processing conditions and resulting composite properties, it was one aim of this study to evaluate the influence of different processing parameters on the residence time of PCL in the extrusion process at a constant temperature profile. A certain degradation and therefore decreased viscosity of PCL during extrusion should not effect the residence time as some authors found experimentally only a low influence of viscosity [28] and others found no effect [29].

\section{Influence of screw elements and $L / D$ of the screw on residence time $t_{R}$}

Figure 5-A illustrates $t_{R}$ of PCL during extrusion in dependence on screw configuration and rotation speed, whereas the last-mentioned parameter was varied on two levels at 100 and $500 \mathrm{~min}^{-1}$. The choice of screw elements like kneading or mixing has a significant influence on the residence time $t_{R}$ of PCL during extrusion. As SC1 contains kneading elements, which are partially designed to be back-conveying, this screw configuration results in longer $t_{R}$ $(+10 \%)$ in comparison to SC3, which contains mixing elements exhibiting a neutral conveying behaviour. This effect was observed for both rotation speeds. The addition of further back-conveying elements in SC2 in comparison to SC1 leads to a significant increase of $t_{R}$ of $10\left(100 \mathrm{~min}^{-1}\right)$ and $30 \%\left(500 \mathrm{~min}^{-1}\right)$. The same trend was found for the screw configurations containing mainly mixing elements (SC4 versus SC3). The biggest effect with an increase in $t_{R}$ of $50 \%$ for both rotation speeds was determined when increasing the extruders processing length to $1200 \mathrm{~mm}$ (L/D 48) in SC5.

\section{Influence of rotation speed and throughput on residence time $t_{R}$}

As illustrated in Figure 5-A increasing rotation speed decreases $t_{R}$ by 25 to $40 \%$ in case of all investigated screw configurations. The presented effect is moderate even if the rotation speed was increased by the factor of five. Vergnes et al. reported a computated dependency of 
residence time vs. rotation speed which fitted an exponential decay [30]. This dependency could not be proofed in this study, as only two data points at 100 and $500 \mathrm{~min}^{-1}$ were collected. Therefore, the plotted lines in Figure 5-A are just a guide for the eyes and are not based on a model assumption. Furthermore, the presented and discussed trends are consistent with experimental data published in [31, 32].

The influence of throughput on $t_{R}$ was investigated in the range between 5 and $15 \mathrm{~kg} / \mathrm{h}$, whereas the rotation speed was kept constant at $500 \mathrm{~min}^{-1}$. Figure 5-B reveals that $t_{R}$ decreases exponentially with increasing throughput for all screw configurations. The reduction of $t_{R}$ is in the range of 50 to $60 \%$ when increasing the throughput from 5 to $15 \mathrm{~kg} / \mathrm{h}$. This finding is again consistent with the simulated data obtained by Vergnes et al. [30] and experimental data published in [31,32].

\subsection{Influence of processing conditions on CNT dispersion in masterbatches}

\section{Influence of rotation speed}

As CNT agglomerates have high cohesive strengths due to van-der-Waals forces and physical entanglements, critical shear stresses have to be exceeded during melt mixing to disperse them. Rotation speed and matrix viscosity are the major parameters to control the shear forces acting on the CNT agglomerates. The increase of rotation speed in twin-screw extrusion of PCL masterbatches containing $7.5 \mathrm{wt} \% \mathrm{CNT}$, while keeping throughput $(5 \mathrm{~kg} / \mathrm{h})$ and temperature profile constant, was found to improve significantly the macroscopic CNT dispersion for all investigated screw configurations. In all cases, aside from SC5, where no masterbatch could be produced with $100 \mathrm{~min}^{-1}$ due to a torque overload, the area fraction $\mathrm{A}_{\mathrm{A}}$ decreased by about $70 \%$ with increasing rotation speed from 100 to $500 \mathrm{~min}^{-1}$ (Figure 6). This finding can be explained with the increasing SME input, which is introduced into the polymer melt during the melt mixing process with higher rotation speed according to Equation 2, although $t_{R}$ decreases by $25-40 \%$ (compare Figure 5-A). Due to a torque reduction at higher rotation speed, caused by shear thinning and a certain thermal degradation of PCL during extrusion, the SME input is tripled instead of quintupled when increasing rotation speed from 100 to $500 \mathrm{~min}^{-1}$. A power law relationship between area fraction $A_{A}$ and SME input was found for both, dispersive and distributive mixing screws (Figure 7). The use of screws containing mixing elements leads to a higher degree of CNT dispersion which can be attributed to the high mixing efficiency of mixing elements.

The size distributions of remaining primary agglomerate for masterbatches processed with screw configurations SC1 and SC2 reveal detailed information about the state of MWNT 
dispersion in dependence on rotation speed (Figure 8). The agglomerate size distribution of the masterbatch produced with SC1 and $100 \mathrm{~min}^{-1}$ contains primary agglomerates with sizes between 10 and $120 \mu \mathrm{m}$, whereas the maximum of the distribution curve is in range of $40 \mu \mathrm{m}$ (Figure 8-A). The use of additional back-conveying elements (SC2) results in decrease of $\mathrm{A}_{\mathrm{A}}$ from 13 to $11 \%$, although the distribution curve is broadened and agglomerates having sizes up to $150 \mu \mathrm{m}$ were detected (Figure 8-B). An increase of rotation speed leads to a reduction of the maximum primary agglomerate size for both screw configurations ( $\mathrm{SC} 1: 120$ to $100 \mu \mathrm{m}$; SC2: 150 to $120 \mu \mathrm{m}$; Figure 8). Furthermore, a significant reduction of primary agglomerates in all size classes was observed.

A significant reduction of $\mathrm{A}_{\mathrm{A}}$ with increasing rotation speed was also found for PCL masterbatches (7.5 wt\% CNT) processed with screw configurations containing mixing elements (SC3-SC5, see Figure 6). Furthermore, the maximum primary agglomerate size increased from 80 to $100 \mu \mathrm{m}$ when increasing the number of back-conveying elements of the screw (SC3 vs. SC4). Interestingly, the maximum primary agglomerate size was with $100 \mu \mathrm{m}$ significantly smaller in comparison to $150 \mu \mathrm{m}$ observed for masterbatches processed with screw configurations containing kneading elements (SC4 vs. SC2). The existence of less big agglomerates is the main reason for the much lower $\mathrm{A}_{\mathrm{A}}$ of masterbatches processed with distributive screw configurations containing mixing elements (SC3-SC5). A further increase of processing length and therefore increased $t_{R}$ from 75 to 115 seconds (SC5) lead only to a minor reduction of $\mathrm{A}_{\mathrm{A}}$.

\section{Influence of throughput}

Besides rotation speed, throughput is an important parameter to control the SME input and residence time during extrusion. Whereas the increase of rotation speed leads to increasing SME input, the increase of throughout in twin-screw extrusion has the opposite effect. The increase of throughput in twin-screw extrusion of PCL masterbatches containing $7.5 \mathrm{wt} \%$ CNT, while keeping rotation speed $\left(500 \mathrm{~min}^{-1}\right)$ and temperature profile constant, was found to decrease the area ratio $A_{A}$ as shown in Figure 9. Again a power law relationship between the area fraction $\mathrm{A}_{\mathrm{A}}$ and $\mathrm{SME}$ input was observed for dispersive and distributive mixing screws. The residence time decreased with throughput variation from 5 to $15 \mathrm{~kg} / \mathrm{h}$ by 50 to $60 \%$ (compare Figure 5-B).

Figure 10 illustrates the agglomerate size distributions at different throughputs for SC1 clearly indicating a significant increase of $\mathrm{A}_{\mathrm{A}}$ in nearly all size classes with increasing throughput (Figure 10A). In comparison to the variation in rotation speed, the width of CNT agglomerate size distribution is now independent of throughput. The maximum CNT 
agglomerate size within the PCL masterbatches is $100 \mu \mathrm{m}$ for all three processing conditions. Furthermore, the number of CNT agglomerates in the range between 70 and $100 \mu \mathrm{m}$

(Figure 10B) remains nearly constant. The differences in $A_{A}$ result from significant reduced agglomerate counts in the range of 10 to $60 \mu \mathrm{m}$, where the particle number is strongly reduced with decreasing throughput. As processing of PCL masterbatches with a throughput of $15 \mathrm{~kg} / \mathrm{h}$ and a rotation speed of $500 \mathrm{~min}^{-1}$ leads to a residence time of only 45 seconds, it can be concluded that large primary agglomerates above $100 \mu \mathrm{m}$ are dispersed very fast when the mixing forces exceed a critical value.

The results concerning the area fraction $\mathrm{A}_{\mathrm{A}}$ in dependence on throughput obtained for masterbatches processed with the screw configurations SC2-SC5 (not shown here) are in accordance with the results described for the use of SC1. Again, a significant reduction of agglomerate numbers was determined for primary CNT agglomerate sizes between 10 and $60 \mu \mathrm{m}$, whereas the number of lager agglomerates above $60 \mu \mathrm{m}$ remained constant with variation of the throughput.

As discussed before, the variation of rotation speed and throughput influences next to the SME also the residence time of PCL during twin-screw extrusion. When plotting the area fraction $\mathrm{A}_{\mathrm{A}}$ versus residence time, an exponential decrease can be observed (Figure 11) for both types of screws.

\section{Morphology development along the extrusion screw}

In order to get an impression how mixing and kneading elements act on the CNT dispersion and where the CNT dispersion takes place, several samples of approximately one gram were taken along the screws behind every mixing or kneading element. Two different processes were investigated using the screw configurations SC2 and SC5 at $5 \mathrm{~kg} / \mathrm{h}$ and $500 \mathrm{~min}^{-1}$. As shown in Figure 12, the area fraction $\mathrm{A}_{\mathrm{A}}$ decreases continuously along the extrusion length. A high dispersion capacity of primary CNT agglomerates was found at the beginning of the extrusion length, whereas the dispersion rate decreases towards the die. As shown in

Figure 13 the width of agglomerate size distribution is not influenced along the extruder, like discussed before for the influence of throughput. The maximum CNT agglomerate size of $120 \mu \mathrm{m}$ observed directly behind the melting zone was also found in the material close to the extrusion die. This finding indicates again that the maximum size of primary CNT agglomerates is not influenced by the extrusion length or residence time. Moreover, it is the particle size distribution, which changes significantly for small agglomerates in the size range between 10 and $60 \mu \mathrm{m}$. It has to be assumed, that the rupture of very big agglomerates to 
fragments of $100 \mu \mathrm{m}$ or smaller happens in the melting zone of the twin-screw extruder at the very beginning of the mixing process.

\subsection{Masterbatch dilution}

The masterbatch processed with screw configuration SC5 using a rotation speed of $500 \mathrm{~min}^{-1}$ and a throughput of $5 \mathrm{~kg} / \mathrm{h}$ exhibited the highest degree of CNT macro dispersion and was therefore diluted with neat polycaprolactone CAPA 6800 in order to generate a percolation set. The processing was performed under the same conditions as the masterbatch production and CNT contents between 0.125 and 4.0 wt.\% were adjusted.

Figure 14 shows light microscopic images of two PCL composites with 0.5 to $1.0 \mathrm{wt}$.\% CNT content, which reveal a nearly perfect macroscopic CNT dispersion within the matrix, as just few very small remaining agglomerates in the micron scale were observed. The measurement of electrical resistivity of PCL based composites filled with MWNT N7000 (Figure 15) indicated a very low percolation threshold. Using a curve fitting procedure above the percolation concentration, the percolation threshold was determined to be $0.37 \mathrm{wt} . \%$, which corresponds to $0.24 \mathrm{vol} \%$. Even if these results were obtained on compression-moulded plates, they illustrate that for future applications, e.g. in melt spun fibres, by using suitable mixing conditions conductive composite materials with good macroscopic dispersion and low nanotube contents can be produced.

\section{Summary and conclusions}

The presented article discusses the influence of extrusion parameters like rotation speed, throughput, and screw configuration on the residence time $t_{R}$ and specific mechanical energy SME input during processing of PCL based masterbatches with carbon nanotubes. Both, residence time and SME value were correlated with the macroscopic CNT dispersion within the PCL matrix, which was quantified in terms of the area fraction $\mathrm{A}_{\mathrm{A}}$ between area occupied by remaining primary agglomerates and investigated area by means of light microscopy on thin sections.

The rotation speed, which mainly controls the shear conditions during extrusion, was found to have a high influence on the CNT macro dispersions in PCL. The determined area fraction $\mathrm{A}_{\mathrm{A}}$ decreased significantly by about $70 \%$ when increasing the rotation speed from 100 to $500 \mathrm{~min}^{-1}$, although a decrease of residence time in the range of 25 to $40 \%$ was observed. This finding was valid for all screw configurations. The much better macro dispersion has therefore to be attributed to the three times higher input of specific mechanical energy. As the area fraction $\mathrm{A}_{\mathrm{A}}$ was found to follow a power law dependency on SME input, it has to be 
assumed, that a further increase of rotation speed does not result in the complete dispersion of CNT in the PCL matrix. Studying the primary MWNT agglomerate size distribution curves revealed that an increase of rotation speed leads to a decrease of the distribution width by 20 to 30 microns and a significant reduction of number of CNT agglomerates of smaller sizes. However, undispersed CNT agglomerates in the range of $100 \mu \mathrm{m}$ were observed even in the masterbatch processed with $500 \mathrm{~min}^{-1}$.

The residence time during extrusion of PCL was found to depend strongly on the throughput, whereas an increase of throughput from 5 to $15 \mathrm{~kg} / \mathrm{h}$ resulted in a decrease of $t_{\mathrm{R}}$ in the order of $50 \%$. Although the variation of throughput had no influence on the width of the agglomerate size distribution of remaining agglomerates, a significant reduction of the agglomerate number of small agglomerates with sizes between 10 and $60 \mu \mathrm{m}$ was observed. The third important processing parameter in twin-screw extrusion is the design of the screw configuration, where two principle design possibilities were studied; extrusion screws with mainly mixing or kneading elements. The investigation of the influence of screw design on $t_{R}$ indicated a significant influence, of the choice of screw elements. As kneading blocks exhibit an active conveying effect, the use of these elements results in longer residence times in comparison to the use of mixing elements. This effect is independent of rotation speed. The further replacement of conveying elements by back-conveying elements leads to a significant increase of the residence time of about 10 to $30 \%$, depending on the applied rotation speed. This trend was found for dispersive and distributive screw configurations. The biggest effect on the residence time was achieved with extending the extruders processing length to $1200 \mathrm{~mm}$ (L/D 48). An increase of residence time of $50 \%$ for both investigated rotation speeds was found.

Although all distributive screw configurations exhibit shorter residence times in comparison to the dispersive pendants, the use of these screws led to significantly better CNT dispersions. This finding can be related to the much higher mixing efficiency of such mixing elements. Furthermore, the mixing elements resulted in significantly lower SME inputs, which can be advantageous in order to minimise the CNT shortening expected to occur during extrusion processes.

Finally, the masterbatch exhibiting best macroscopic CNT dispersion was diluted under the same processing conditions. The area fraction $\mathrm{A}_{\mathrm{A}}$ of this masterbatch was determined to be $2.2 \%$, whereas these agglomerates were completely dispersed in the dilution process.

Compression-moulded samples of these composites showed a very low percolation threshold of 0.37 wt. $\%$ which corresponds to 0.24 vol. $\%$. 
The paper is a first attempt to understand the relationship between extrusion conditions and morphology of composites containing carbon nanotubes and summarizes experimental findings. Even if more investigations are needed it represents a basic for further comprehension of the underlying basic dispersion processes.

\section{Acknowledgement}

We gratefully acknowledge financial support from the project INTELTEX (Intelligent multireactive textiles integrating nanofiller based CPC-fibres) - a European Integrated Project supported through the Sixth Framework Programme for Research and Technological development and to Nanocyl S.A. for providing the nanotubes within the INTELTEX project. In addition, we are grateful to José A. Covas (University of Minho, Guimarães, Portugal) for the helpful discussions about the interpretation of extrusion experiments.

\section{References}

1. Iijima S, Helical Microtubules of Graphitic Carbon, Nature, 1991;354(6348):56-58.

2. Saito R, Fujita M, Dresselhaus G and Dresselhaus MS, Electronic-Structure of Chiral Graphene Tubules, Applied Physics Letters, 1992;60(18):2204-2206.

3. Wildoer JWG, Venema LC, Rinzler AG, Smalley RE and Dekker C, Electronic structure of atomically resolved carbon nanotubes, Nature, 1998;391(6662):59-62.

4. Odom TW, Huang JL, Kim P and Lieber CM, Atomic structure and electronic properties of single-walled carbon nanotubes, Nature, 1998;391(6662):62-64.

5. Berber S, Kwon YK and Tomanek D, Unusually high thermal conductivity of carbon nanotubes, Physical Review Letters, 2000;84(20):4613-4616.

6. Kim P, Shi L, Majumdar A and McEuen PL, Thermal transport measurements of individual multiwalled nanotubes, Physical Review Letters, 2001;8721(21):

7. Che JW, Cagin T and Goddard WA, Thermal conductivity of carbon nanotubes, Nanotechnology, 2000;11(2):65-69.

8. Wong EW, Sheehan PE and Lieber CM, Nanobeam mechanics: Elasticity, strength, and toughness of nanorods and nanotubes, Science, 1997;277(5334):1971-1975.

9. Treacy MMJ, Ebbesen TW and Gibson JM, Exceptionally high Young's modulus observed for individual carbon nanotubes, Nature, 1996;381(6584):678-680.

10. Yakobson BI, Brabec CJ and Bernholc J, Nanomechanics of carbon tubes: Instabilities beyond linear response, Physical Review Letters, 1996;76(14):2511-2514.

11. Krause B, Petzold G, Pegel S and Pötschke P, Correlation of carbon nanotube dispersability in aqueous surfactant solutions and polymers, Carbon, 2009;47(3):602-612.

12. Kasaliwal G, Göldel A and Pötschke P, Influence of Processing Conditions in SmallScale Melt Mixing and Compression Molding on the Resistivity and Morphology of Polycarbonate-MWNT Composites, Journal of Applied Polymer Science, 2009;112(6):34943509 .

13. Krause B, Pötschke P and Häussler L, Influence of small scale melt mixing conditions on electrical resistivity of carbon nanotube-polyamide composites, Composites Science and Technology, 2009;69(10):1505-1515.

14. Pegel S, Pötschke P, Petzold G, Alig I, Dudkin SM and Lellinger D, Dispersion, agglomeration, and network formation of multiwalled carbon nanotubes in polycarbonate melts, Polymer, 2008;49(4):974-984. 
15. Villmow T, Pötschke P, Pegel S, Häussler L and Kretzschmar B, Influence of twinscrew extrusion conditions on the dispersion of multi-walled carbon nanotubes in a poly(lactic acid) matrix, Polymer, 2008;49(16):3500-3509.

16. Villmow T, Pegel S, Pötschke P and Wagenknecht U, Influence of injection molding parameters on the electrical resistivity of polycarbonate filled with multi-walled carbon nanotubes, Composites Science and Technology, 2008;68(3-4):777-789.

17. Lellinger D, Xu DH, Ohneiser A, Skipa T and Alig I, Influence of the injection moulding conditions on the in-line measured electrical conductivity of polymer-carbon nanotube composites, Physica Status Solidi B-Basic Solid State Physics, 2008;245(10):22682271.

18. Yuan JM, Fan ZF, Chen XH, Chen XH, Wu ZJ and He LP, Preparation of polystyrene-multiwalled carbon nanotube composites with individual-dispersed nanotubes and strong interfacial adhesion, Polymer, 2009;50(14):3285-3291.

19. Chen L, Pang XJ and Yu ZL, Study on polycarbonate/multi-walled carbon nanotubes composite produced by melt processing, Materials Science and Engineering a-Structural Materials Properties Microstructure and Processing, 2007;457(1-2):287-291.

20. Xu GY, Du LC, Wang H, Xia R, Meng XC and Zhu QR, Nonisothermal crystallization kinetics and thermomechanical properties of multiwalled carbon nanotubereinforced poly(epsilon-caprolactone) composites, Polymer International, 2008;57(9):10521066.

21. Wu D, Wu L, Sun Y and Zhang M, Rheological properties and crystallization behavior of multi-walled carbon Nanotube/Poly(epsilon-caprolactone) composites, Journal of Polymer Science Part B-Polymer Physics, 2007;45(23):3137-3147.

22. Saeed K and Park SY, Preparation and properties of multiwalled carbon nanotube/polycaprolactone nanocomposites, Journal of Applied Polymer Science, 2007;104(3):1957-1963.

23. Mitchell CA and Krishnamoorti R, Dispersion of single-walled carbon nanotubes in poly(epsilon-caprolactone), Macromolecules, 2007;40(5):1538-1545.

24. Shaffer MSP and Windle AH, Fabrication and characterization of carbon nanotube/poly(vinyl alcohol) composites, Advanced Materials, 1999;11(11):937-941.

25. Rwei SP, Manas-Zloczower I and Feke DL, Characterization of Agglomerate Dispersion by Erosion in Simple Shear Flows, Polymer Engineering and Science, 1991;31(8):558-562.

26. Kasaliwal GR, Pegel S, Göldel A, Pötschke P and Heinrich G, Analysis of agglomerate dispersion mechanisms of multiwalled carbon nanotubes during melt mixing in polycarbonate, Polymer, 2010;51(12):2708-2720.

27. Pötschke P, Dudkin SM and Alig I, Dielectric spectroscopy on melt processed polycarbonate--multiwalled carbon nanotube composites, Polymer, 2003;44(17):5023-5030. 28. Altomare RE and Ghossi P, An Analysis of Residence Time Distribution Patterns in a Twin Screw Cooking Extruder, Biotechnology Progress, 1986;2(3):157-163.

29. Kao SV and Allison GR, Residence Time Distribution in a Twin Screw Extruder, Polymer Engineering and Science, 1984;24(9):645-651.

30. Poulesquen A and Vergnes B, A study of residence time distribution in co-rotating twin-screw extruders. Part I: Theoretical modeling, Polymer Engineering and Science, 2003;43(12):1841-1848.

31. Poulesquen A, Vergnes B, Cassagnau P, Michel A, Carneiro OS and Covas JA, A study of residence time distribution in co-rotating twin-screw extruders. Part II: Experimental validation, Polymer Engineering and Science, 2003;43(12):1849-1862.

32. Puaux JP, Bozga G and Ainser A, Residence time distribution in a corotating twinscrew extruder, Chemical Engineering Science, 2000;55(9):1641-1651. 


\section{Tables}

Table 1: Temperature profiles for masterbatch processing; extrusion direction: 1 (hopper) to 10 (die)

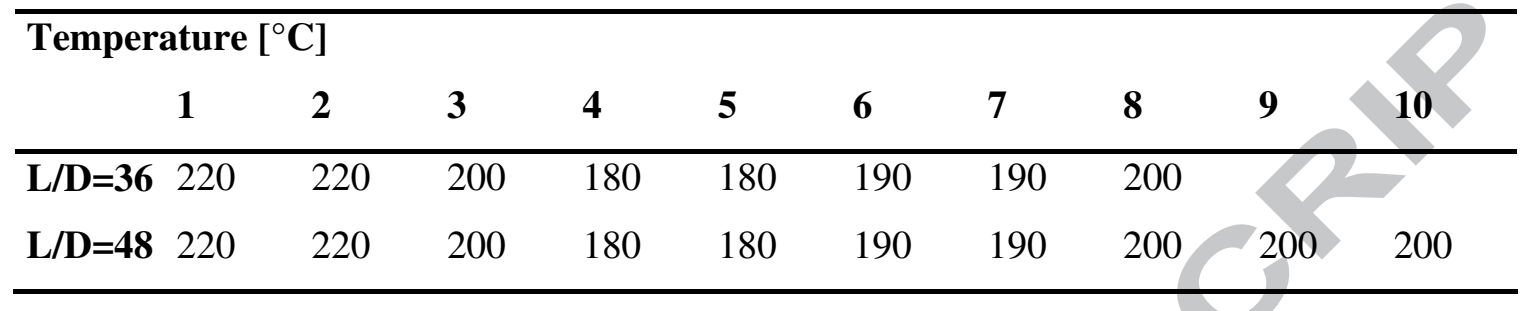

Table 2: Specification of extrusion screw elements used for masterbatch processing corresponding to Fig. 2

\begin{tabular}{|c|c|c|c|c|c|c|c|}
\hline No. & $\begin{array}{l}\text { length } \\
{[\mathrm{mm}]}\end{array}$ & $\begin{array}{l}\text { pitch } \\
{[\mathrm{mm}]}\end{array}$ & $\begin{array}{l}\text { no. of } \\
\text { flights }\end{array}$ & $\begin{array}{c}\text { type of element } \\
\text { m- mixing } \\
\text { k- kneading } \\
\text { c- conveying }\end{array}$ & $\begin{array}{l}\text { no. of } \\
\text { discs } \\
\text { (no. of } \\
\text { cogs) }\end{array}$ & $\begin{array}{c}\text { staggering } \\
\text { angle }\left[{ }^{\circ}\right]\end{array}$ & $\begin{array}{c}\text { conveying } \\
\text { behaviour: } \\
\text { L- left handed } \\
\text { R- right handed }\end{array}$ \\
\hline 1 & 37.5 & 37.5 & 2 & $\mathrm{c}$ & & & $\mathrm{L}$ \\
\hline 2 & 25.0 & 25.0 & & $\mathrm{c}$ & & & $\mathrm{L}$ \\
\hline 3 & 12.5 & 25.0 & & $\mathrm{c}$ & & & $\mathrm{R}$ \\
\hline 4 & 25.0 & & & $\mathrm{k}$ & 5 & 45 & $\mathrm{~L}$ \\
\hline 5 & 25.0 & & & $\mathrm{k}$ & 5 & 45 & $\mathrm{R}$ \\
\hline 6 & 37.5 & & & $\mathrm{k}$ & 5 & 90 & \\
\hline 7 & & & & $\mathrm{~m}$ & $3(10)$ & & \\
\hline 8 & 15.0 & & & $\mathrm{~m}$ & $2(10)$ & & \\
\hline
\end{tabular}




\section{Figures}

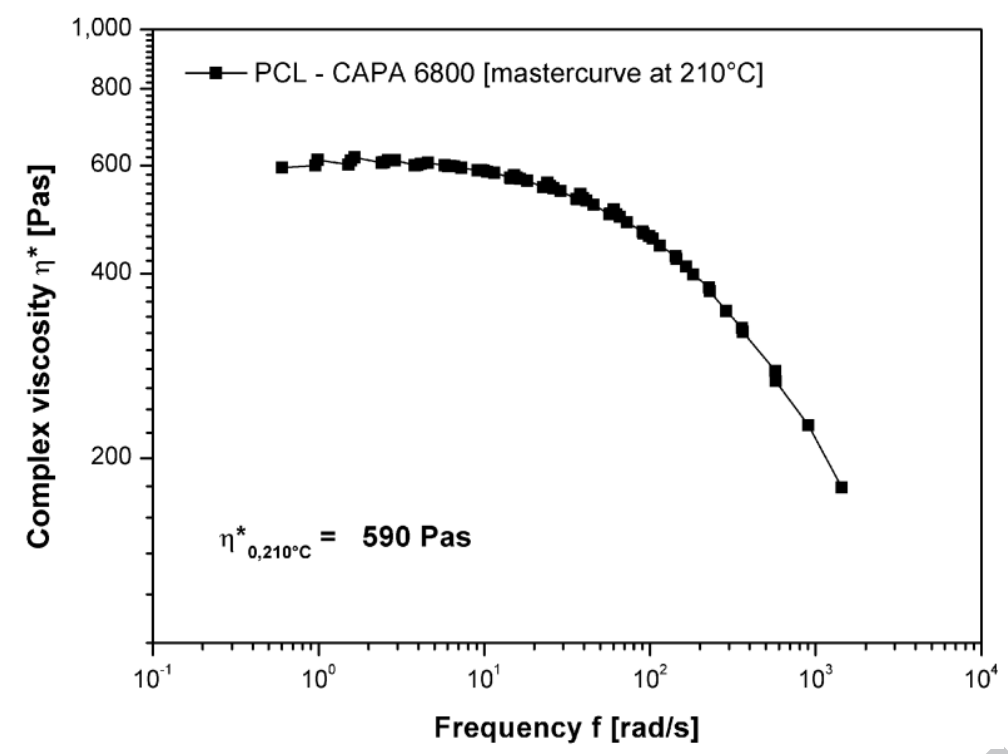

Figure 1: Frequency dependent viscosity of PCL CAPA 6800 at $210^{\circ} \mathrm{C}$ (mastercurve based on measurements at $150^{\circ} \mathrm{C}, 180^{\circ} \mathrm{C}$, and $210^{\circ} \mathrm{C}$ using an Ares oscillation rheometer)

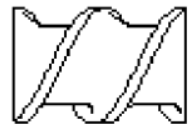

1

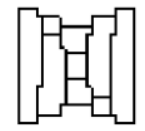

5

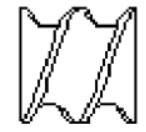

2

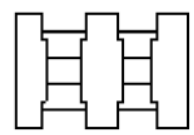

6

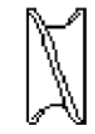

3

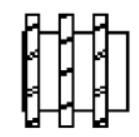

7

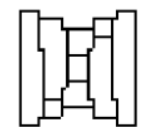

4

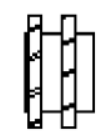

8

Figure 2: Screw elements for twin-screw extruder ZE25 from Berstorff; conveying elements $(1,2)$, back-conveying element (3), kneading elements (4-6), mixing elements $(7,8)$ 
SC-1

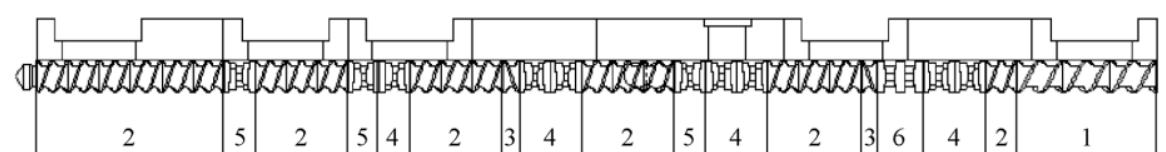

SC-2

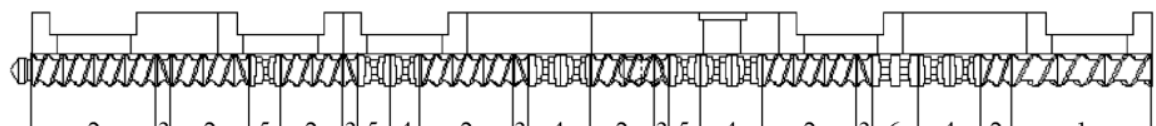

SC-3

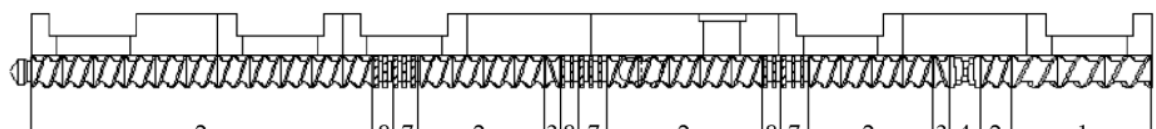

SC-5

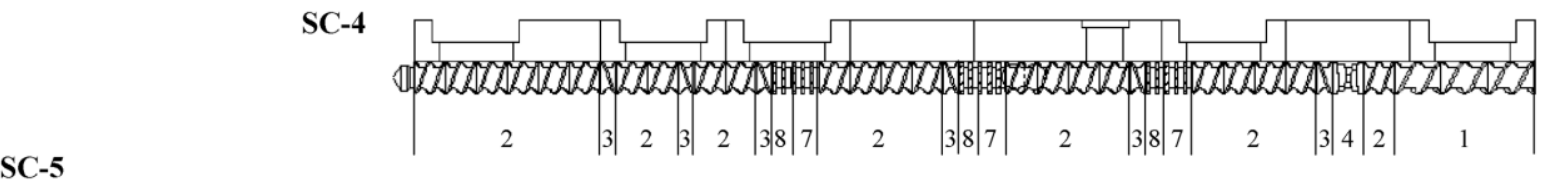

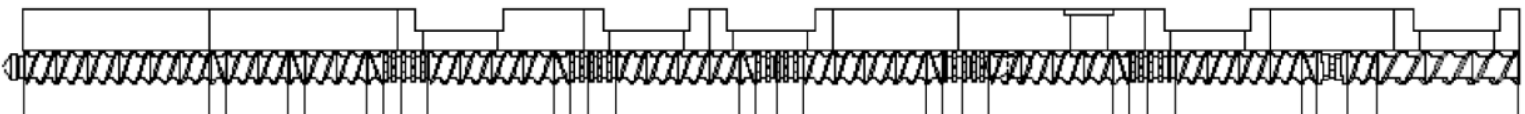

Figure 3: Modular extrusion screw configurations based on individual screw elements
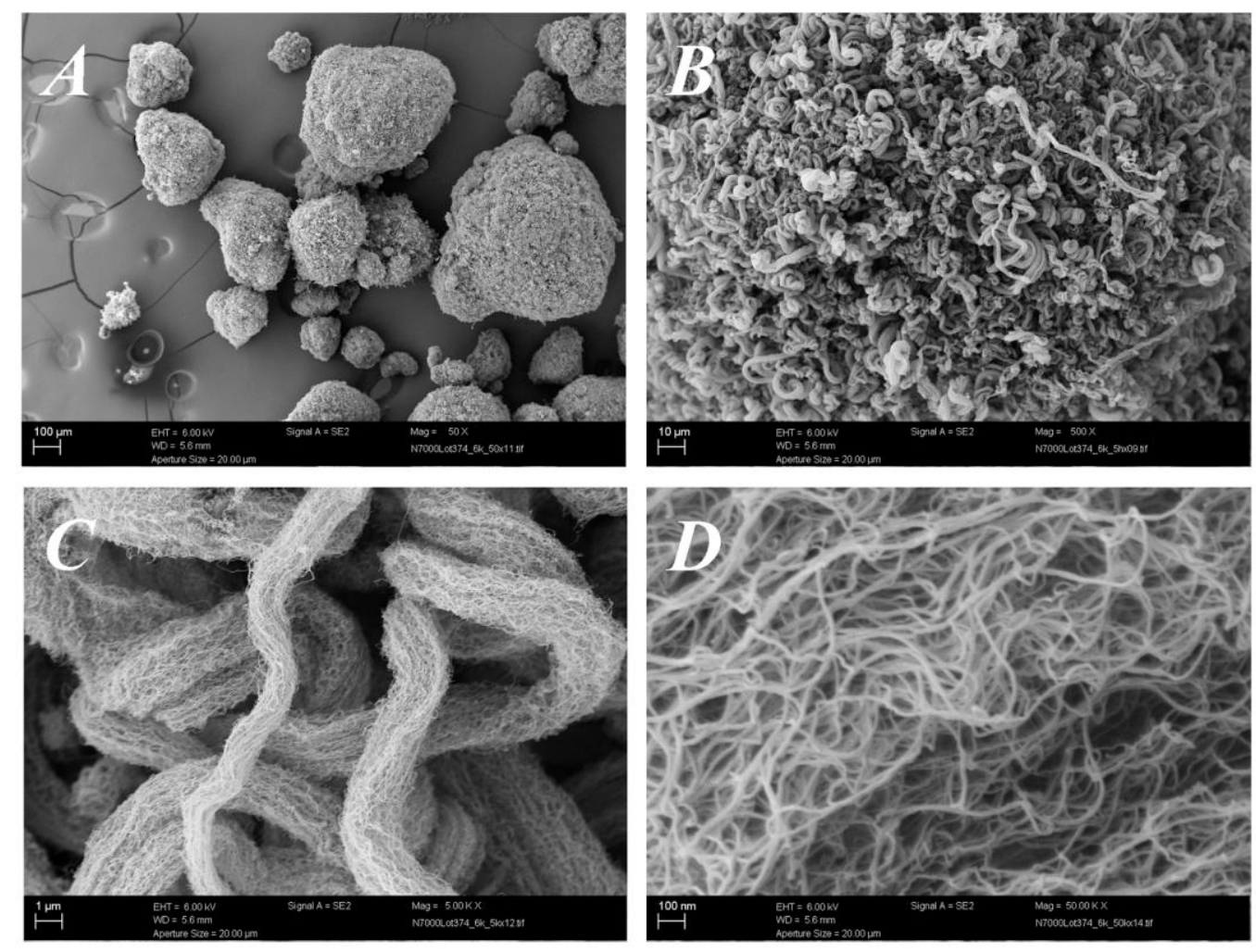

Figure 4: SEM micrographs of CNT N7000 raw material at different magnifications 

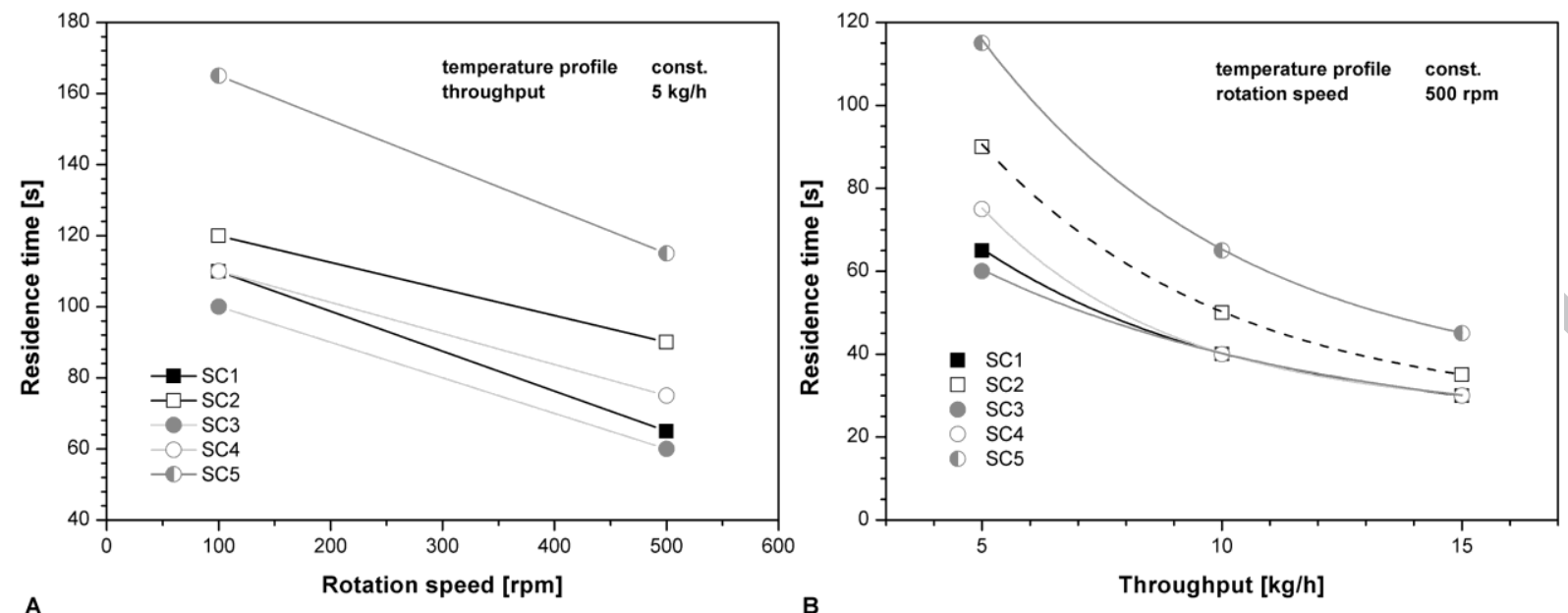

Figure 5: Residence time of PCL depending on screw configuration and processing parameters; A) rotation speed, B) throughput

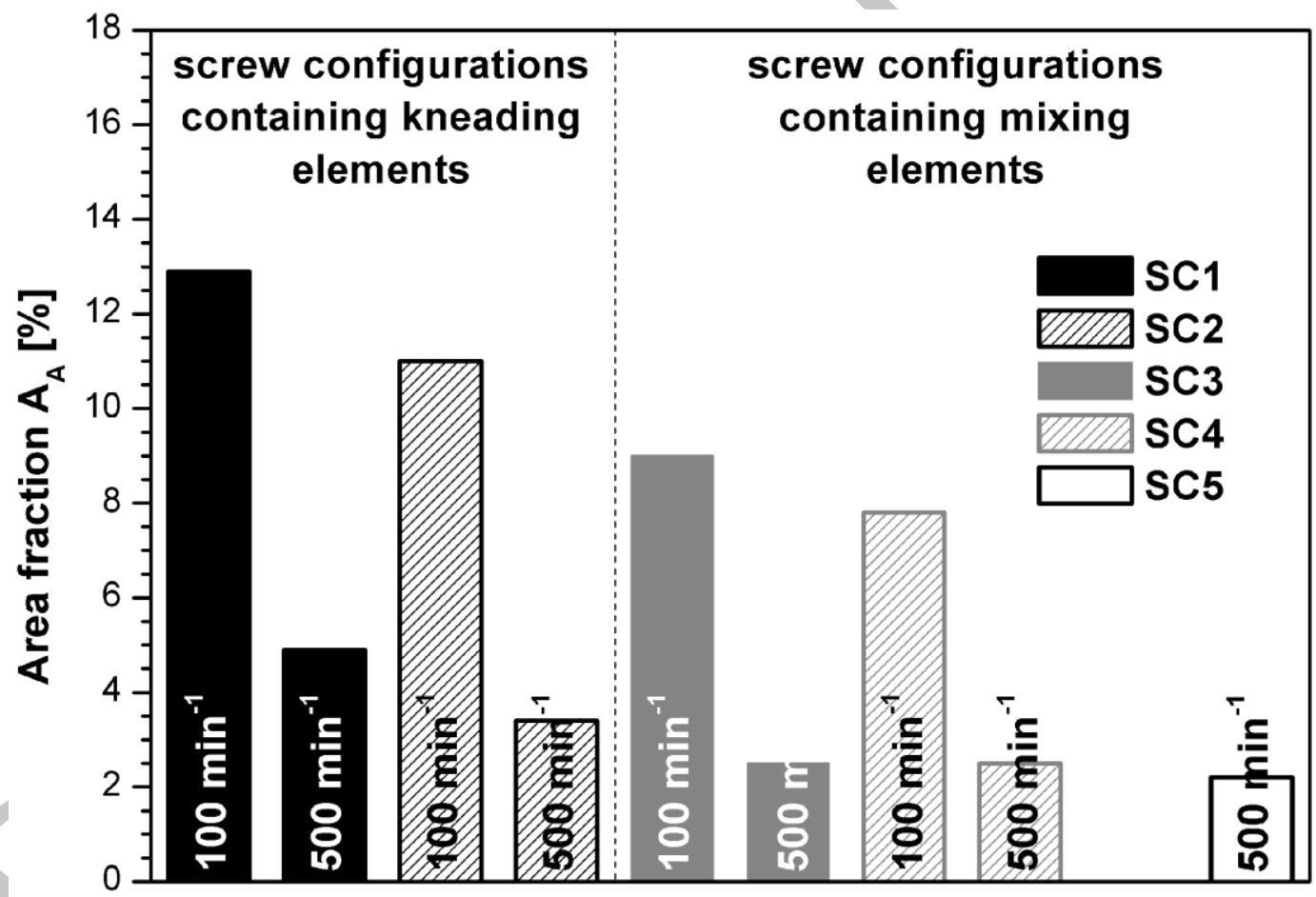

Figure 6: Area fraction $A_{A}$ determined for thin sections of PCL based masterbatches with 7.5 wt.\% CNT depending on rotation speed and screw configuration 


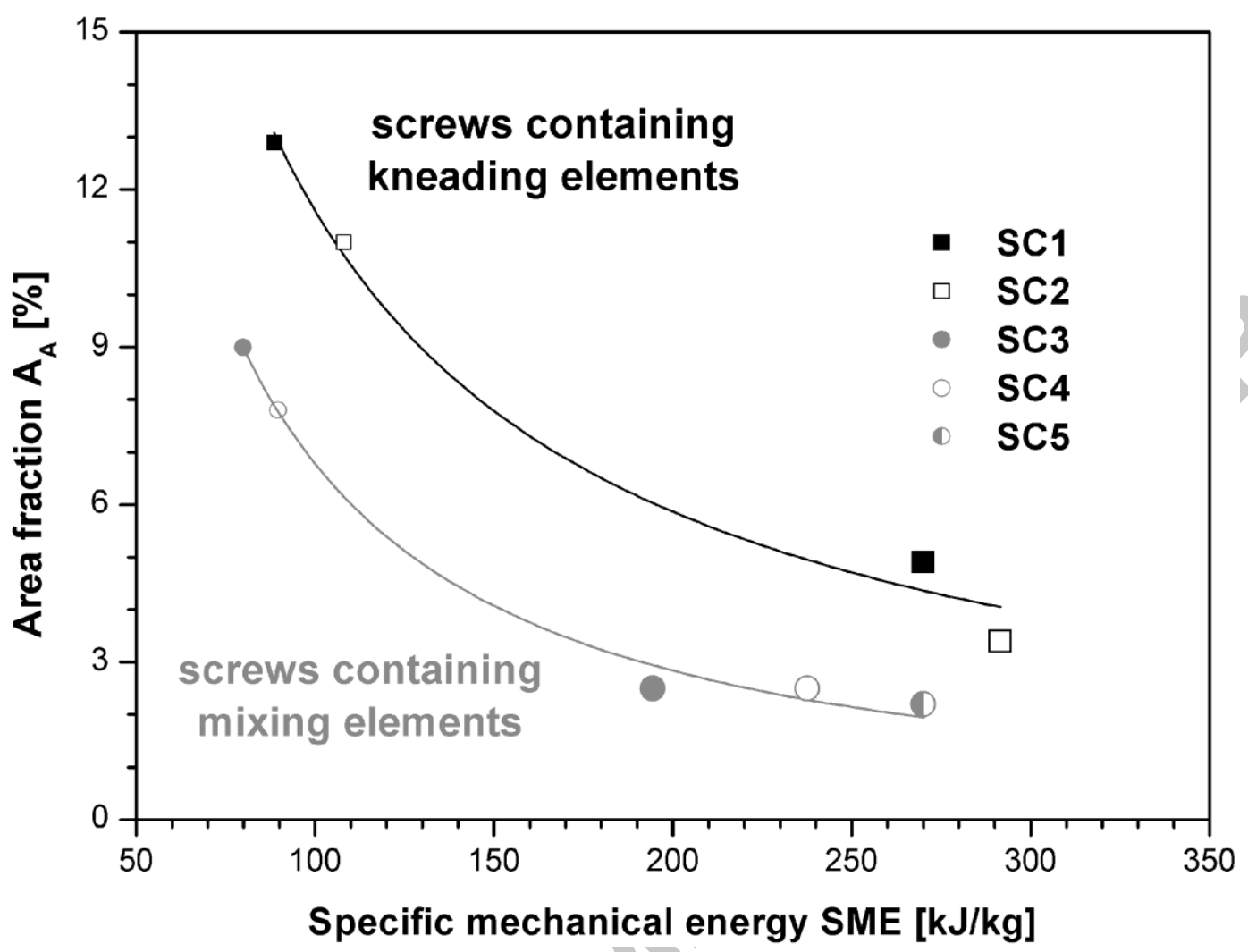

Figure 7: Area fraction $\mathrm{A}_{\mathrm{A}}$ determined for thin sections of PCL based masterbatches with 7.5 wt.\% CNT depending on specific mechanical energy SME (variation of rotation speed, throughput: $5 \mathrm{~kg} / \mathrm{h}$, increasing rotation speed is indicated by increasing size of symbols)
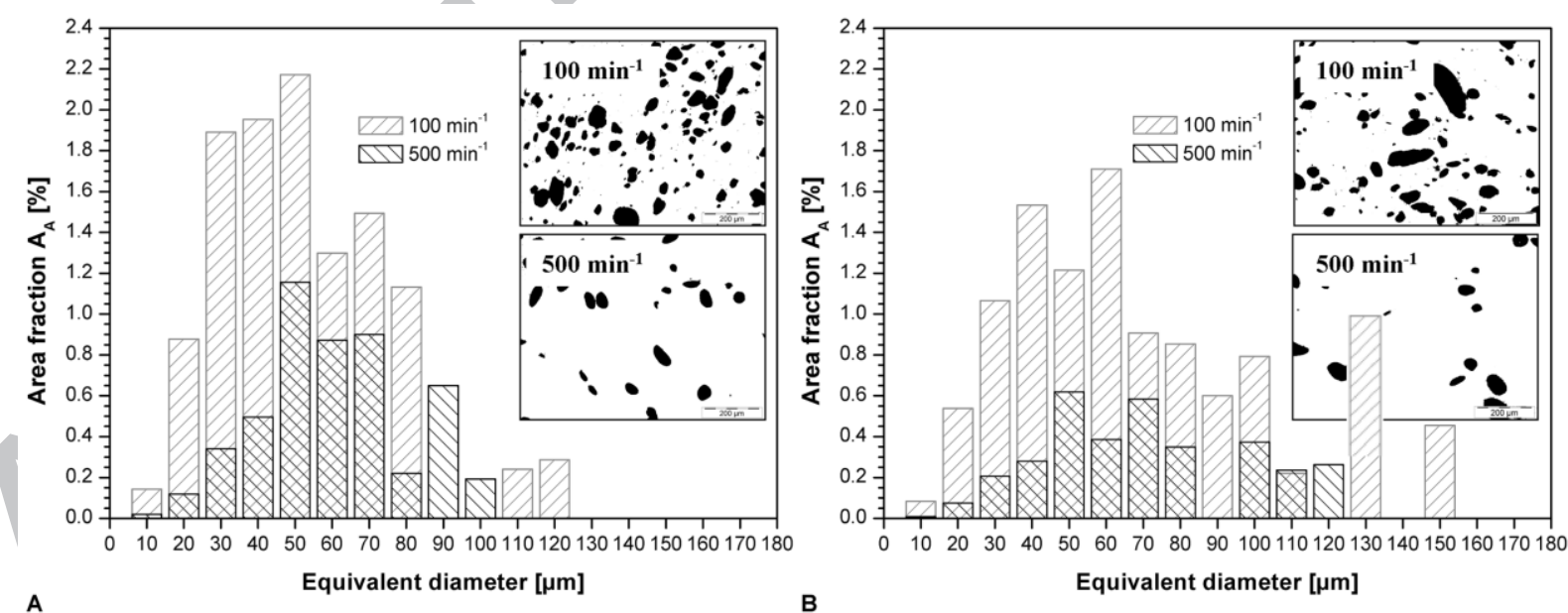

Figure 8: CNT agglomerate size distributions of PCL based masterbatches with 7.5 wt.\% CNT depending on rotation speed, A: SC1, B: SC2 


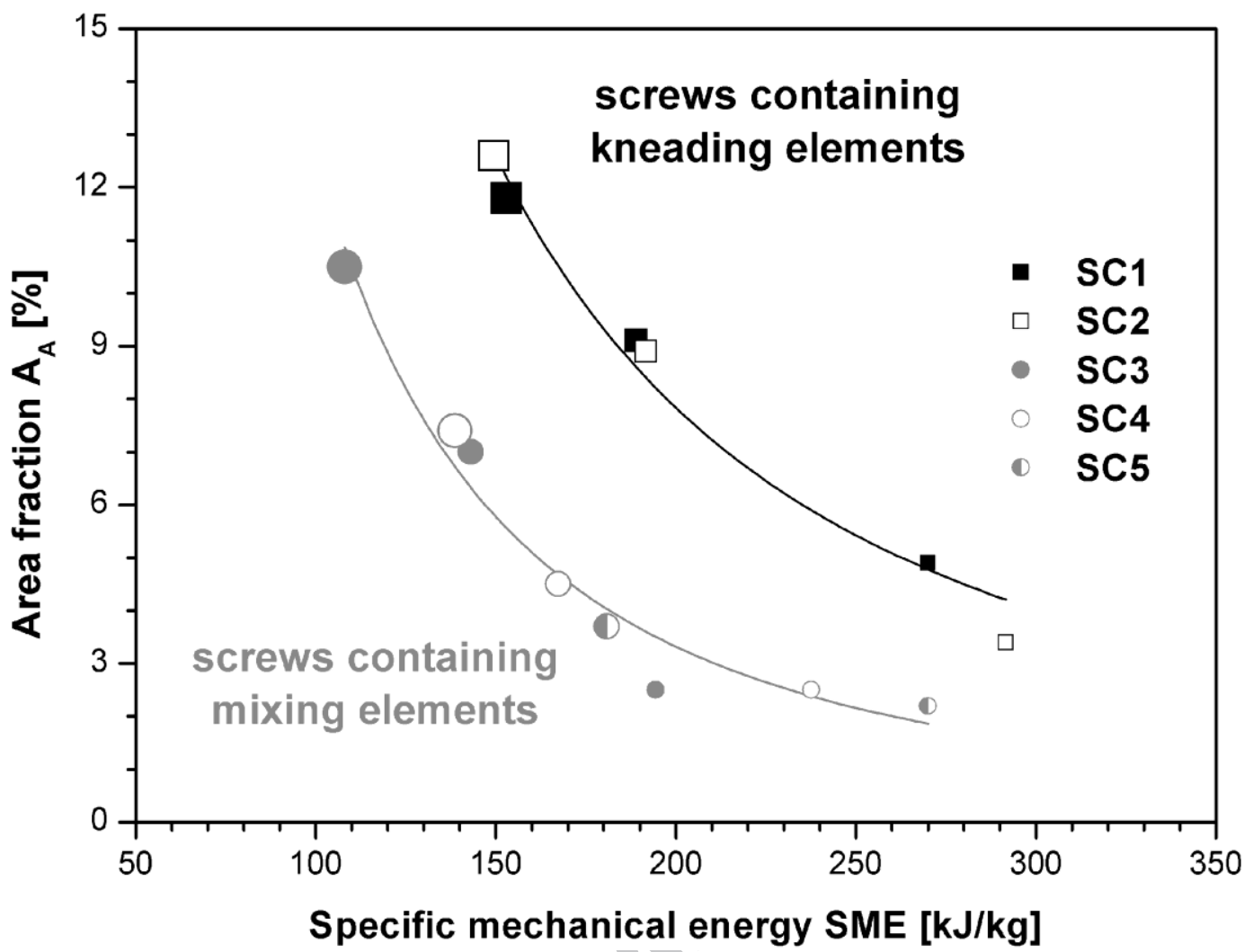

Figure 9: Area fraction $A_{A}$ determined for thin sections of PCL based masterbatches with 7.5 wt.\% CNT depending on specific mechanical energy SME (variation of throughput, rotation speed: $500 \mathrm{~min}^{-1}$, increasing throughput is indicated by increasing size of symbols)
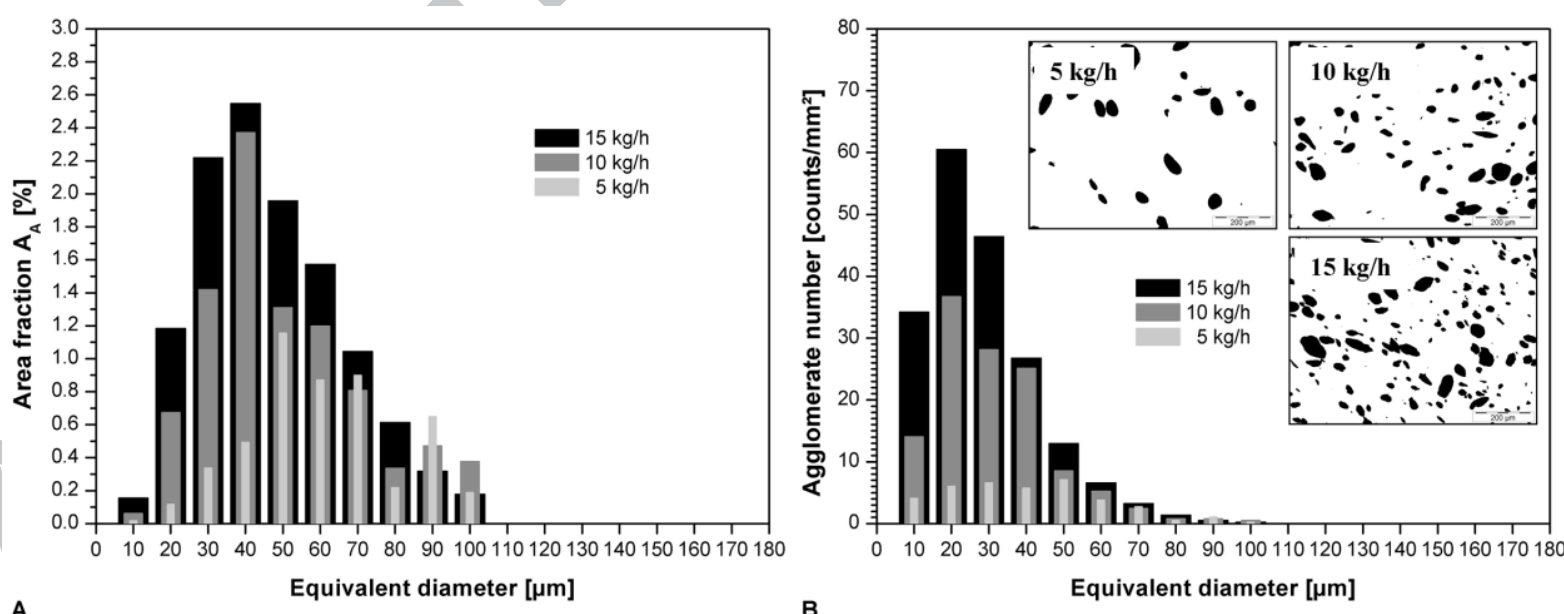

Figure 10: CNT agglomerate size distributions of PCL based masterbatch with 7.5 wt.\% CNT (SC1) depending on throughput, A: area fraction $\mathrm{A}_{\mathrm{A}}, \mathrm{B}$ : agglomerate number 


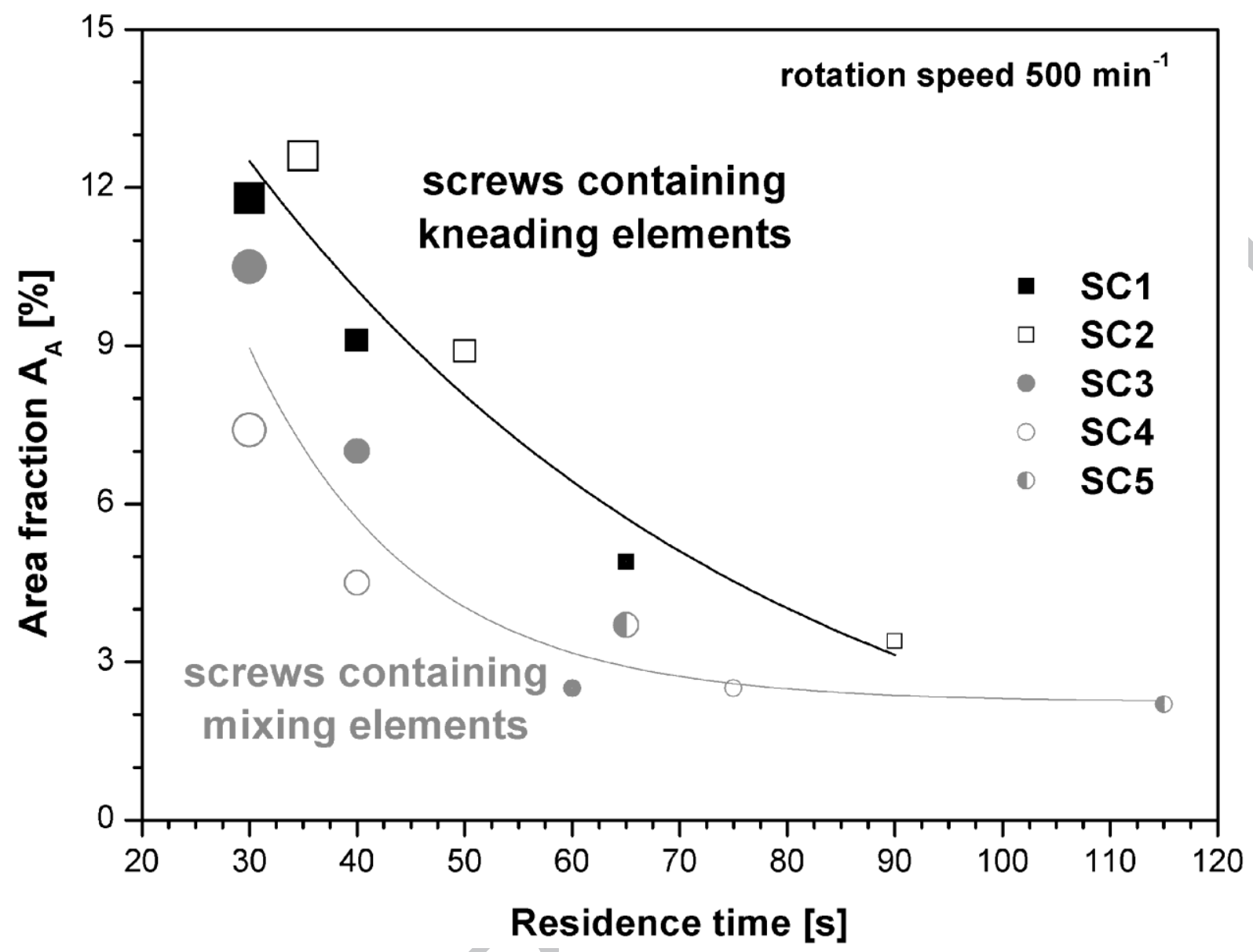

Figure 11: Area fraction $A_{A}$ determined for thin sections of PCL based masterbatches with 7.5 wt.\% CNT depending on residence time (variation of throughput, rotation speed: $500 \mathrm{~min}^{-}$ ${ }^{1}$, increasing throughput is indicated by increasing size of symbols) 


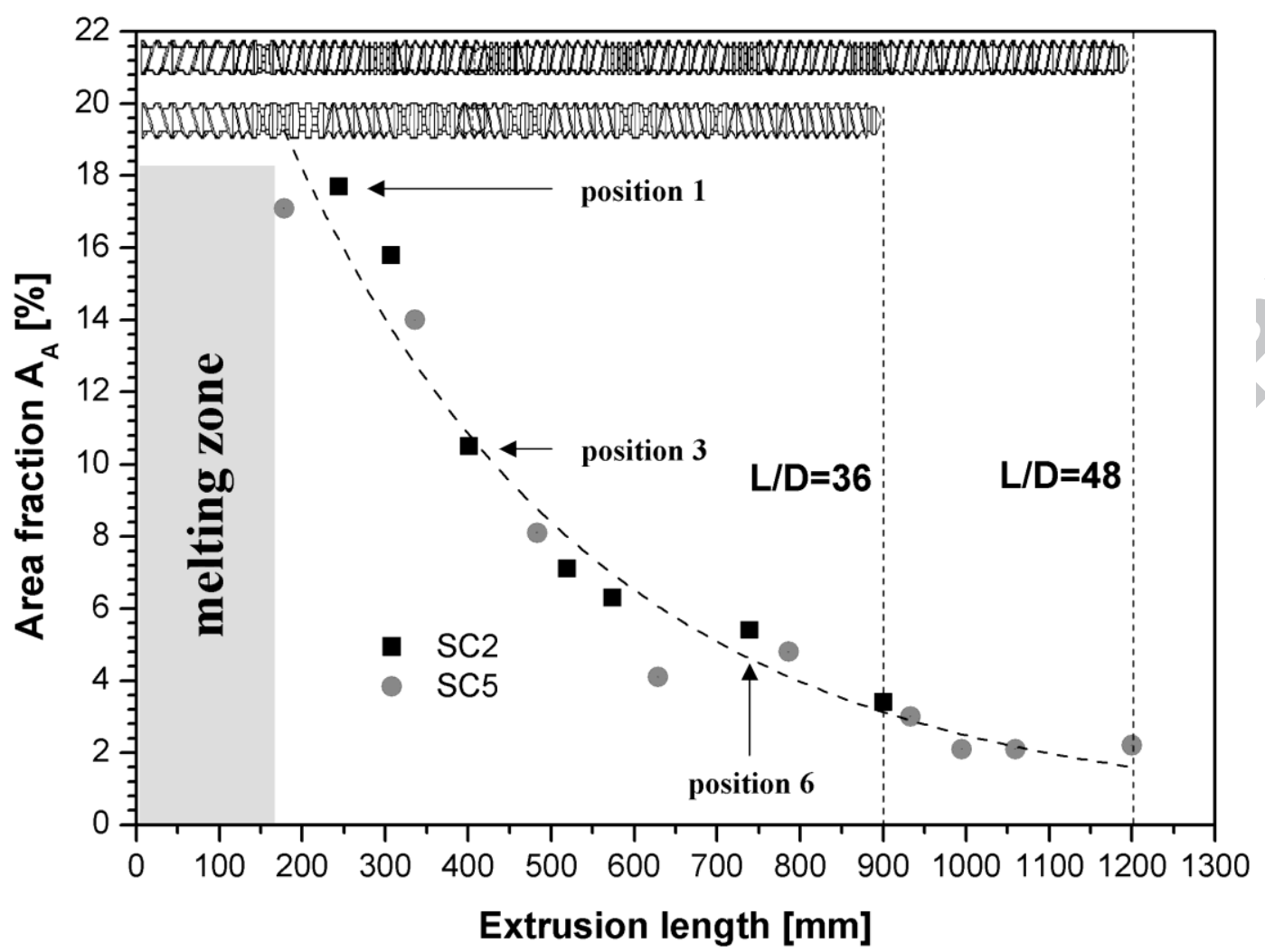

Figure 12: Development of area fraction $A_{A}$ determined for thin sections of PCL based masterbatches with 7.5 wt.\% CNT along the extrusion length for SC2 and SC5

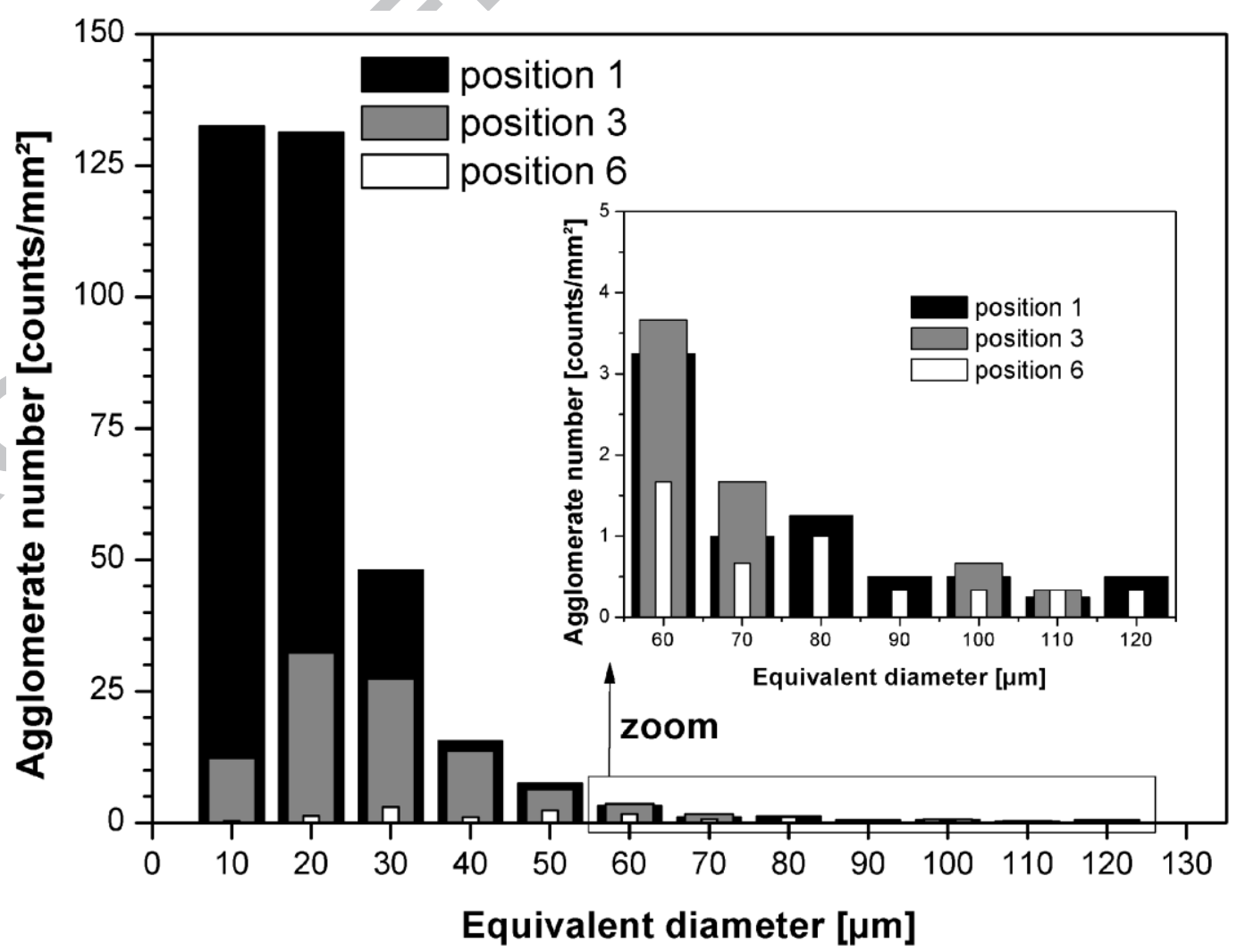

Figure 13: Development of CNT agglomerate size distribution along the extrusion length of PCL based masterbatches (7.5 wt.\% CNT) processed with screw configuration SC2 

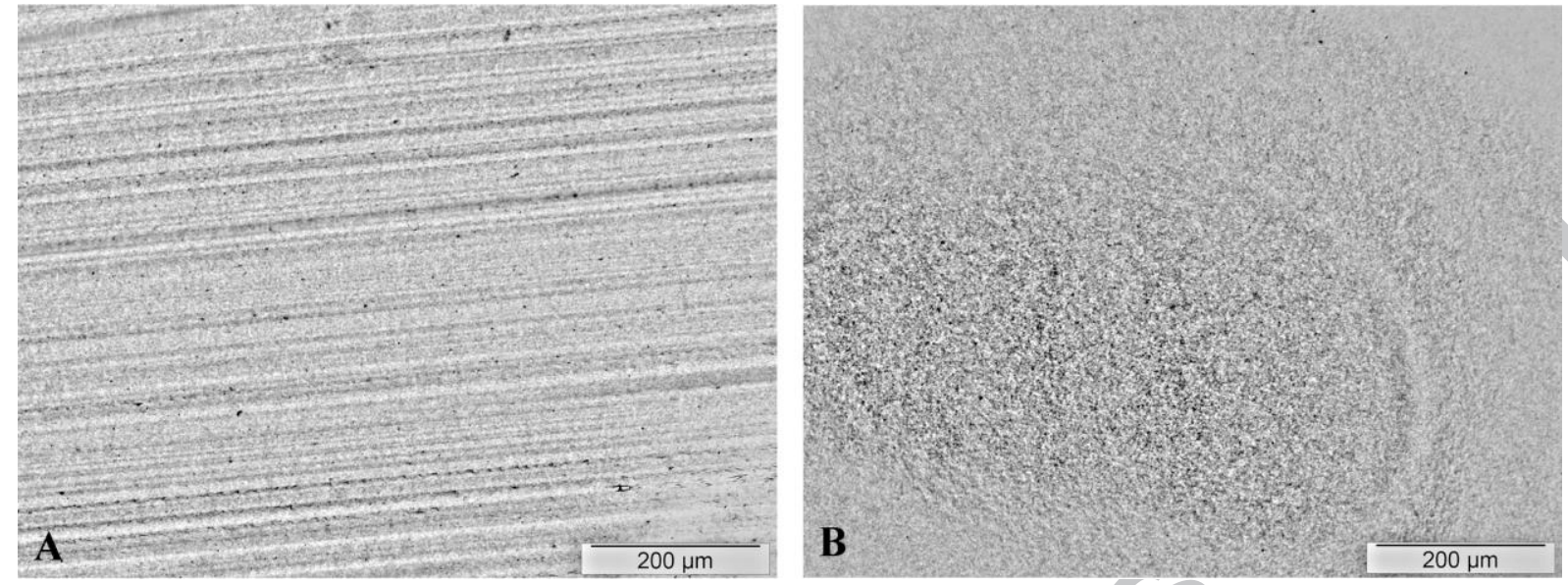

Figure 14: Light microscopic images of PCL composites with different CNT contents;

A: 0.5 wt. $\%$, B: 1.0 wt.\%

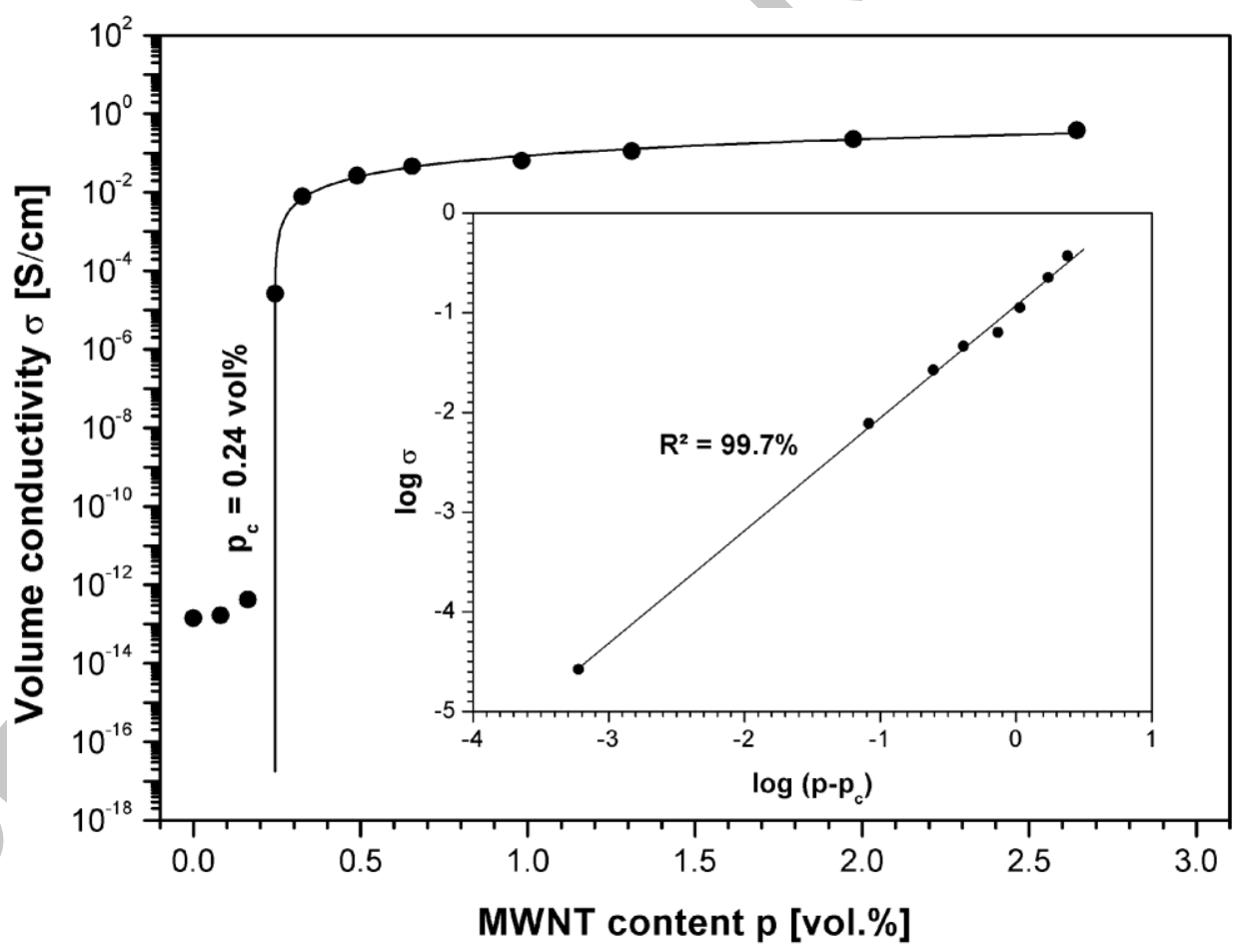

Figure 15: Electrical percolation behaviour of MWNT N7000 in PCL based composites 